\title{
Personal Identity and Patient-Centered Medical Decision Making
}

Lucie White

\section{Pre-print version. Published (2017) in AJOB Neuroscience, 8(3): 194-195. http://dx.doi.org/10.1080/21507740.2017.1366590}

Nancy Jecker and Andrew Ko (2017) wish to present an account of personal identity which captures what matters to the patient and places the patient at the center of medical decisions. They focus particularly on medical interventions in the brain that can cause drastic changes in personality; under what circumstances should we say the patient has 'survived' these changes? More specifically, how can we best understand the notion of survival in a way that captures what is of concern to the patient? This goal is laudable, however, their chosen account of narrative identity is ill-suited to this task for one reason in particular; it does not give sufficient guidance in predicting which medical decisions are likely to be experienced as disruptive to identity.

According to Jecker and Ko's formulation of narrative identity, psychological elements, personality traits and other attributes are properly attributable to the person insofar as they are incorporated into "the self-told story of his or her life" (2017, p.4). Though a person's values and traits may form reference points in one's autobiographical narrative, proponents of narrative identity emphasize that a person is not constituted by her values and psychological contents, but by her story. Making a decision that fits the narrative, therefore, should be regarded as distinct from acting upon one's settled values and beliefs (Blustein 1999). When a patient wants to know whether and to what extent they will 'survive' a medical intervention, and we endorse a narrative account of identity, we must therefore ask, to what extent could any anticipated changes be incorporated into the patient's self-told narrative?

It should not be thought that the patient is free to construct her narrative in an arbitrary fashion. Narrative identity is subject to some important constraints. Jecker and Ko stress the condition of 'correctness'. This seems akin to Marya Schechtman's 'reality constraint'; the requirement that a narrative must correspond with basic observational facts about the world (Baylis 2009). Other narrative theorists highlight a condition of 'coherence', although it's not clear precisely to what standard a narrative should be held (Blustein 1999). 
Even with these constraints, it is clear that narrative identity is compatible with radical change (Baylis 2009). Jecker and Ko take the dynamic nature of narrative identity to be one of its key strengths. But it presents us with a problem in a medical context; it does not provide an indication of which changes will or could be incorporated seamlessly into the patient's narrative, and which might be experienced as troubling and disruptive to one's identity. The condition of incorporation into a narrative is very unlikely ever to point us in a clear direction; several alternatives are likely to fit the narrative equally well whenever a choice is faced (Kuczewski 1999). Schechtman notes that narratives can be made intelligible even when they are based around fundamental transformations that one strongly repudiates, or that are caused by oppressive social structures (2003).

A narrative account of identity, then, does not give sufficient guidance concerning the future to enable a prediction concerning how the outcome will be experienced by the agent. This is compounded by Jecker and Ko's focus on narrative repair. This activity is necessarily retrospective - it must be conducted after a significant change has already taken place. The narrative account of identity thus does not adequately address what Jecker and Ko identify as the central issue concerning personal identity: what is of concern to the patient when he asks whether he will survive a brain-altering procedure in a meaningful sense.

An account of personal identity that sees psychological connectedness (or continuity) ${ }^{1}$ as the basis of personal identity gives us better grounds for predicting what effects and side effects of brain altering surgery are likely to be experienced as disruptive to personal identity, and thus as threatening to survival in the sense that Jecker and Ko are concerned with. At the same time, it is just as patient-centered as Jecker and Ko's account. These notions of personal identity see the patient's deeply held, enduring desires, values, goals, and other psychological elements as constitutive of the patient's personal identity. If we understand personal identity in this way, the concern is that a radical disruption of the psychological elements that constitute the self disturbs the psychological connections between the patient before the intervention and afterwards, in a way that is not voluntarily embraced by the agent, given her existing motivational set.

This approach to personal identity captures what concerns the patient when he asks whether he will survive some therapy that potentially involves drastic personality changes. When a patient fears

\footnotetext{
${ }^{1}$ A sufficiently robust account of continuity might be the best way to approach personal identity on a global level, throughout the course of the patient's entire life. But faced with a certain decision, it may be better to focus on connectedness; to what extent do the existing psychological elements that constitute the patient remain, and to what extent are they disrupted or replaced? For more on this distinction, see Parfit (1971).
} 
that he will not survive a procedure, that is, that the resulting person will be someone else, he fears that the deeply held, enduring values, beliefs and desires which form the core of his self will be replaced by others, and will no longer have the ability to motivate him (Schechtman 2003). A focus on psychological connectedness also helps us to assess, before the fact, which potential changes to personality may be experienced as disruptive to identity. Potential alterations to personality must be assessed on the basis of the enduring psychological elements that form the basis of the patient's deepest self. When these changes are concordant with or endorsable on the basis of these values, beliefs and desires, the alteration is unlikely to be experienced as disruptive to identity. When the changes are at odds with the patient's existing motivational set, or particularly when changes may alter the patient's motivational set to the extent that his previous values and beliefs no longer figure into his decision-making process, survival may be threatened.

This account gives physician and patient a means of proceeding with a decision concerning the types of interventions that Jecker and Ko focus upon. The physician must, in conversation with the patient, carefully gauge the enduring values, beliefs and desires that form the basis of the patient's self, and use this to identify which anticipated changes potentially form a threat to identity. Discussion can then proceed around these identified areas of concern. Where a side effect appears unexpectedly, these conversations can be used to determine how to best proceed (whether, for example, the change needs to be carefully reviewed by the patient with DBS switched off).

A focus on the existing values of the patient as the basis for personal identity captures considerations advanced by others in the field, particularly in the context of DBS. Sven Nyholm and Elizabeth O'Neill note that whether the effects of DBS are experienced as alien will be deeply influenced by the agent's values; if an agent values spontaneity, they might see an increased tendency to act on one's impulses as a welcomed and potentially authenticity-increasing side effect of DBS; whereas if these tendencies are disvalued, they will be more likely to be experienced as disruptive for identity (2016). Francoise Baylis notes that some commentators focus exclusively on unwanted side effects of DBS when discussing potential threats to personal identity (2009).

Jecker and Ko are right to draw our attention to accounts of personal identity that are focused on survival, construed in a way that is centered around patient concerns, rather than an abstract, metaphysical notion of numerical identity. In order to achieve their commendable goals, however, they would be better off endorsing a notion of identity that sees the values, goals and beliefs that the patient holds dear as constitutive of the patient. Such an account literally puts what matters to 
the patient at the center of considerations regarding personal identity, and can be used to better ensure that the concerns of the patient form the basis of decision-making in this domain.

\section{References}

Baylis, F. 2009. "I am who I am": on the perceived threats to personal identity from deep brain stimulation. Neuroethics 6(3): 513-526.

Blustein, J. 1999. Choosing for others as continuing a life story: the problem of personal identity revisited. Journal of Law, Medicine and Ethics, 27(1): 32-36.

Jecker, N. and A. Ko. 2017. Is that the same person? The problem of identity following neurosurgical procedures. AJOB Neuroscience, 8(3): 2-29.

Kuczewski, M. 1999. Commentary: narrative views of personal identity and substituted judgment in surrogate decision making. Journal of Law, Medicine and Ethics, 27(1): 32-36.

Nyholm, S. and E. O’Neill. 2016. Deep brain stimulation, continuity over time, and the true self. Cambridge Quarterly of Healthcare Ethics, 25(4): 647-58.

Parfit, D. 1971. Personal identity. The Philosophical Review, 80(1): 3-27.

Schechtman, M. 2003. Empathic access: the missing ingredient in personal identity. In Personal Identity, ed. R. Martin and J. Barresi, 238-59, Oxford: Blackwell. 